\title{
Insulinoma-associated protein 1 (INSM1) is a sensitive and highly specific marker of neuroendocrine differentiation in primary lung neoplasms: an immunohistochemical study of 345 cases, including 292 whole-tissue sections
}

\author{
Sanjay Mukhopadhyay $\mathbb{D}^{1} \cdot$ Josephine K. Dermawan ${ }^{1} \cdot$ Christopher P. Lanigan $^{1} \cdot$ Carol F. Farver $^{1}$
}

Received: 20 May 2018 / Revised: 24 July 2018 / Accepted: 28 July 2018 / Published online: 28 August 2018

(c) United States \& Canadian Academy of Pathology 2018

\begin{abstract}
Recent evidence suggests a role for the nuclear marker INSM1 in the diagnosis of neuroendocrine lung neoplasms. The aim of this study was to determine the utility of INSM1 as a marker of neuroendocrine differentiation using a large series of whole-tissue sections of primary lung neoplasms. We stained 345 primary lung neoplasms with INSM1, including 292 whole-tissue sections. Most cases were also stained with synaptophysin, chromogranin, and CD56. The tumors included 64 small cell lung carcinomas, 24 large cell neuroendocrine carcinomas, 64 carcinoid tumors (48 typical, 16 atypical), 130 adenocarcinomas, and 33 squamous cell carcinomas. For small cell lung carcinoma, the sensitivity of INSM1 (98\%) was similar to synaptophysin (100\%) and CD56 (95\%) but considerably higher than chromogranin (83\%). For large cell neuroendocrine carcinoma, CD56 (92\%) and synaptophysin $(88 \%)$ were more sensitive than INSM1 (75\%), while chromogranin was less sensitive (46\%). All markers stained $100 \%$ of carcinoid tumors, except one atypical carcinoid tumor, which was negative for INSM1. The sensitivity of INSM1 for neuroendocrine lung neoplasms as a group (95\%) was similar to synaptophysin (98\%) and CD56 (97\%), but higher than chromogranin (84\%). The specificity of INSM1 for neuroendocrine lung neoplasms (97\%) was similar to chromogranin (98\%) but higher than synaptophysin (90\%) and CD56 (87\%). INSM1 staining was concordant in primary tumors and matched metastases. In conclusion, INSM1 is a reliable marker of neuroendocrine differentiation in primary lung neoplasms, with sensitivity similar to synaptophysin and CD56, and specificity similar to chromogranin.
\end{abstract}

\section{Introduction}

Insulinoma-associated protein 1 (INSM1) is a zinc-finger transcription factor that plays an important role in the development of neuroendocrine differentiation in various tissues [1-10]. It is encoded by the insulinoma associated1 (IA-1) gene, the cDNA of which was first identified in 1992 at the National Institutes of Health (Bethesda, MD) by Yasuhiro Goto and colleagues in human pancreatic insulinoma tissue and murine insulinoma cell lines [1]. Goto et al. were also the first to demonstrate expression of IA-1 in small cell lung carcinoma by Northern analysis, as

Sanjay Mukhopadhyay

mukhops@ccf.org

1 Department of Pathology, Cleveland Clinic, Cleveland, OH, USA well as in other neuroendocrine neoplasms, such as pheochromocytoma, medullary thyroid carcinoma, insulinoma, and pituitary tumors. In a subsequent study using human lung cancer cell lines, IA-1 was shown to be expressed in small cell lung carcinoma and carcinoid tumor but not in non-small cell lung carcinoma [2]. The IA-1 gene was subsequently localized to the short arm of chromosome 20 [4].

In the past 3 years, following a seminal report by Rosenbaum and colleagues in 2015 [11], INSM1 has emerged as a robust immunohistochemical marker of neuroendocrine differentiation in normal and neoplastic human tissue, and is currently the only nuclear neuroendocrine marker available for use in diagnostic immunohistochemistry [10-20]. INSM1 expression in lung neoplasms has not been extensively studied except in two recent series, one that used mainly tissue microarrays [14] and another that utilized cytology cell blocks [19]. 
Table 1 Primary lung neoplasms and specimen types used in this study

\begin{tabular}{lllll}
\hline & $\begin{array}{l}\text { No. of } \\
\text { cases }\end{array}$ & $\begin{array}{l}\text { Whole-tissue sections } \\
\text { of resected tumor }(\%)\end{array}$ & $\begin{array}{l}\text { Small biopsies } \\
(\%)\end{array}$ & $\begin{array}{l}\text { Tissue microarray } \\
(\%)\end{array}$ \\
\hline $\begin{array}{l}\text { Small cell lung carcinoma } \\
\text { Large cell neuroendocrine }\end{array}$ & 64 & $32(50)$ & $32(50)$ & $0(0)$ \\
carcinoma & 24 & $24(100)$ & $0(0)$ & $0(0)$ \\
Typical carcinoid tumor & 48 & $42(88)$ & $4(8)$ & $2(4)$ \\
Atypical carcinoid tumor & 16 & $16(100)$ & $0(0)$ & $0(0)$ \\
Neuroendocrine & 152 & $114(75)$ & $36(24)$ & $2(1)$ \\
Adenocarcinoma & 130 & $123(95)$ & $0(0)$ & $7(5)$ \\
Squamous cell carcinoma & 33 & $27(82)$ & $0(0)$ & $6(18)$ \\
Large cell carcinoma & 4 & $4(100)$ & $0(0)$ & $0(0)$ \\
Sarcomatoid carcinoma & 8 & $8(100)$ & $0(0)$ & $0(0)$ \\
Adenosquamous carcinoma & 3 & $3(100)$ & $0(0)$ & $0(0)$ \\
Adenoid cystic carcinoma & 7 & $7(100)$ & $0(0)$ & $0(0)$ \\
Mucoepidermoid carcinoma & 5 & $5(100)$ & $0(0)$ & $0(0)$ \\
Epithelial-myoepithelial & 1 & $0(0)$ & $1(100)$ & $0(0)$ \\
carcinoma & & & & $1(100)$ \\
Sclerosing pneumocytoma & 1 & $0(0)$ & $0(0)$ & $0(0)$ \\
Hamartoma & 1 & $1(100)$ & $2(1)$ & $13(0)$ \\
Non-neuroendocrine & 193 & $178(92)$ & $38(11)$ & $15(4)$ \\
Total & 345 & $292(85)$ & &
\end{tabular}

The aim of this study was to determine the utility of INSM1 as a marker of neuroendocrine differentiation in primary lung neoplasms using a large series of whole-tissue sections of resected tumors. We also sought to investigate INSM1 expression in lung tumors that have not been previously tested with this marker, and to determine whether INSM1 expression in metastases is similar to INSM1 status in matched primaries or is lost in the process of tumor progression and metastasis.

\section{Materials and methods}

\section{Case selection}

This study was approved by the Cleveland Clinic Institutional Review Board (IRB) for Protection of Human Subjects (IRB 17-101). Three hundred and forty-five primary lung neoplasms were retrieved from the archives of the Department of Pathology, Cleveland Clinic, and stained with a mouse monoclonal antibody to INSM1 (clone A-8), including 152 neuroendocrine tumors and 193 nonneuroendocrine tumors (Table 1). Most cases were also stained with synaptophysin, chromogranin, and CD56. The cohort also included primary lung tumors whose INSM1 status has not been previously reported, including large cell carcinoma, adenosquamous carcinoma, and sarcomatoid carcinoma. In most cases $(292 / 345,85 \%)$, wholetissue sections of resected tumors were used for immunohistochemistry, the remainder being small lung biopsies $(38 / 345,11 \%)$ or tissue microarrays $(15 / 345,4 \%)$.

All tumors were diagnosed using 2015 World Health Organization criteria [21]. For small cell lung carcinoma, immunohistochemistry was used at the time of the original diagnosis in $45(70 \%)$ cases, and $19(30 \%)$ were diagnosed without the aid of neuroendocrine markers. In the former group, all tumors were positive for at least one neuroendocrine marker. By definition, all large cell neuroendocrine carcinomas had neuroendocrine morphologic features on $H \& E$, and were positive for at least one neuroendocrine marker.

\section{Immunohistochemistry}

Details of the antibodies used are provided in Table 2. INSM1 was diluted 1:250 using SignalStain ${ }^{\circledR}$ Diluent (Cell Signaling Technology, Danvers, MA) and incubated at ambient temperature for $48 \mathrm{~min}$. Automated staining was performed using a VENTANA Discovery XT platform and RUO XT OptiView DAB IHC v4 software (Ventana Medical Systems, Inc., Tucson, AZ). Staining was detected using the OptiView DAB IHC Detection Kit and amplification (Ventana Medical Systems Inc., Tucson, AZ). Antigen retrieval was performed using cell conditioning solution 2 (CC2, Ventana Medical Systems, Inc., Tucson, AZ) for 32 min. The Universal Linker and horseradish peroxidase multimer were selected for $8 \mathrm{~min}$ each, and the OptiView Amplifier and Amplification multimer were selected for 4 
Table 2 Antibodies used in this study

Table 3 INSM1, synaptophysin, chromogranin, and CD56 in primary lung neoplasms

\begin{tabular}{lllll}
\hline & INSM1 & Synaptophysin & Chromogranin A & CD56 \\
\hline Clone & A-8 & Snp88 & DAK-A3 & MRQ-42 \\
Species & Mouse & Mouse & Mouse & Rabbit \\
$\begin{array}{l}\text { Dilution } \\
\text { Catalog }\end{array}$ & $1: 250$ & Predilute & $1: 100$ & Predilute \\
number & & AM363-5M & M0869 & 156R-98 \\
Clonality & Monoclonal & Monoclonal & Monoclonal & Monoclonal \\
$\begin{array}{l}\text { Positive } \\
\text { control }\end{array}$ & Pancreas & Pancreas & Pancreas & Tonsil \\
Source & Santa Cruz & Biogenex, Fremont, & Agilent, Santa Clara, Cell Marque Corp., \\
& Biotechnology, Inc., & CA & CA & Rocklin, CA \\
& Dallas, TX & & & \\
\hline
\end{tabular}

\begin{tabular}{|c|c|c|c|c|}
\hline \multirow[t]{2}{*}{ Tumor type } & \multicolumn{4}{|c|}{ Positive/total $(\%)$} \\
\hline & INSM1 & Synaptophysin & Chromogranin & CD56 \\
\hline Small cell lung carcinoma & $63 / 64(98)$ & $64 / 64(100)$ & $53 / 64(83)$ & $61 / 64(95)$ \\
\hline Large cell neuroendocrine carcinoma & $18 / 24(75)$ & $21 / 24(88)$ & $11 / 24(46)$ & $22 / 24(92)$ \\
\hline Typical carcinoid tumor & 48/48 (100) & 48/48 (100) & $45 / 45(100)$ & $42 / 42(100)$ \\
\hline Atypical carcinoid tumor & $15 / 16(94)$ & $16 / 16(100)$ & $16 / 16(100)$ & $16 / 16(100)$ \\
\hline All neuroendocrine & $144 / 152(95)$ & $147 / 150(98)$ & $125 / 149(84)$ & $141 / 146(97)$ \\
\hline Adenocarcinoma & $4 / 130(3)$ & $3 / 24(13)$ & $1 / 82(1)$ & $7 / 82(9)$ \\
\hline Squamous cell carcinoma & $1 / 33(3)$ & $1 / 18(6)$ & $0 / 33(0)$ & $6 / 33(18)$ \\
\hline Large cell carcinoma $^{\mathrm{a}}$ & $0 / 4(0)$ & Not tested & $0 / 1(0)$ & $0 / 1(0)$ \\
\hline Sarcomatoid carcinoma $^{a}$ & $0 / 8(0)$ & Not tested & $0 / 1(0)$ & $1 / 1(100)$ \\
\hline Adenosquamous carcinoma $^{a}$ & $0 / 3(0)$ & Not tested & $1 / 2(50)$ & $1 / 3(33)$ \\
\hline Adenoid cystic carcinoma $^{a}$ & $0 / 7(0)$ & Not tested & $0 / 1(0)$ & $1 / 1(100)$ \\
\hline Mucoepidermoid carcinoma $^{\mathrm{a}}$ & $0 / 5(0)$ & Not tested & Not tested & Not tested \\
\hline Epithelial-myoepithelial carcinoma $^{a}$ & $0 / 1(0)$ & Not tested & Not tested & Not tested \\
\hline Sclerosing pneumocytoma ${ }^{a}$ & $0 / 1(0)$ & Not tested & Not tested & Not tested \\
\hline Hamartoma $^{\mathrm{a}}$ & $0 / 1(0)$ & Not tested & $0 / 1(0)$ & $0 / 1(0)$ \\
\hline All non-neuroendocrine & $5 / 193(3)$ & $4 / 42(10)$ & $2 / 121(2)$ & $16 / 122(13)$ \\
\hline
\end{tabular}

${ }^{a}$ Primary lung tumors whose INSM1 status has not been previously reported min each. The VENTANA OptiView DAB IHC Detection Kit was also used for synaptophysin, chromogranin, and CD56 staining, with the BenchMark ULTRA automated slide staining system (Ventana Medical Systems, Inc., Tucson, AZ).

\section{Interpretation of immunohistochemical findings}

Any nuclear staining for INSM1 was interpreted as positive. For synaptophysin and chromogranin, any granular cytoplasmic staining was considered positive. Cytoplasmic or membrane staining was considered positive for CD56. Staining intensity was graded semiquantitatively as weak, moderate, or strong. In all INSM1-positive cases, the percentage of positive tumor cells was recorded.

\section{Results}

\section{INSM1 staining in neuroendocrine lung neoplasms}

The immunohistochemical findings are summarized in Table 3. INSM1 was positive in $144 / 152$ (95\%) neuroendocrine lung neoplasms, including 63/64 (98\%) small cell lung carcinomas, 18/24 (75\%) large cell neuroendocrine carcinomas, 48/48 (100\%) typical carcinoid tumors, and 15/16 (94\%) atypical carcinoid tumors.

Of the 63 INSM1-positive small cell lung carcinomas, staining was strong in 37 and weak to moderate in 26 (Fig. 1). As shown in Table 4, in most small cell lung carcinomas the majority of tumor cells were positive; however, in $10(16 \%)$ cases, staining was very focal $(\leq 10 \%)$. In crushed or necrotic cells, INSM1 staining tended 
Fig. 1 INSM1 and traditional neuroendocrine markers in neuroendocrine lung neoplasms. Morphologic features (a-c, hematoxylin-eosin), INSM1 (df), synaptophysin (g-i), chromogranin $(\mathbf{j}-\mathbf{l})$, and CD56 $(\mathbf{m}-\mathbf{o})$. All images at original magnification $\times 200$. SCLC small cell lung carcinoma, LCNEC large cell neuroendocrine carcinoma, SYN synaptophysin, CHR chromogranin
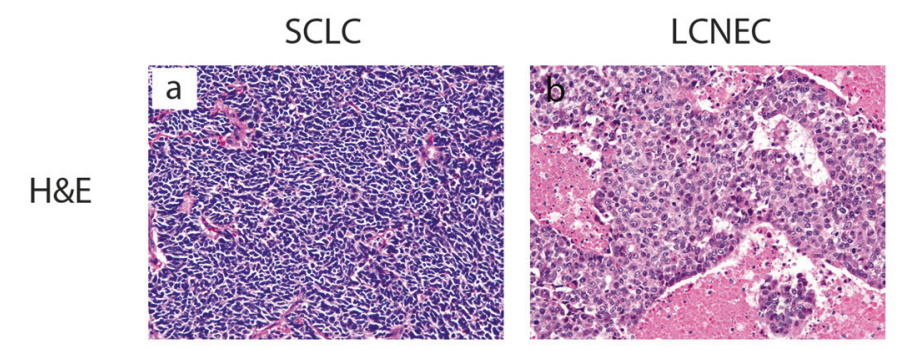

Typical carcinoid
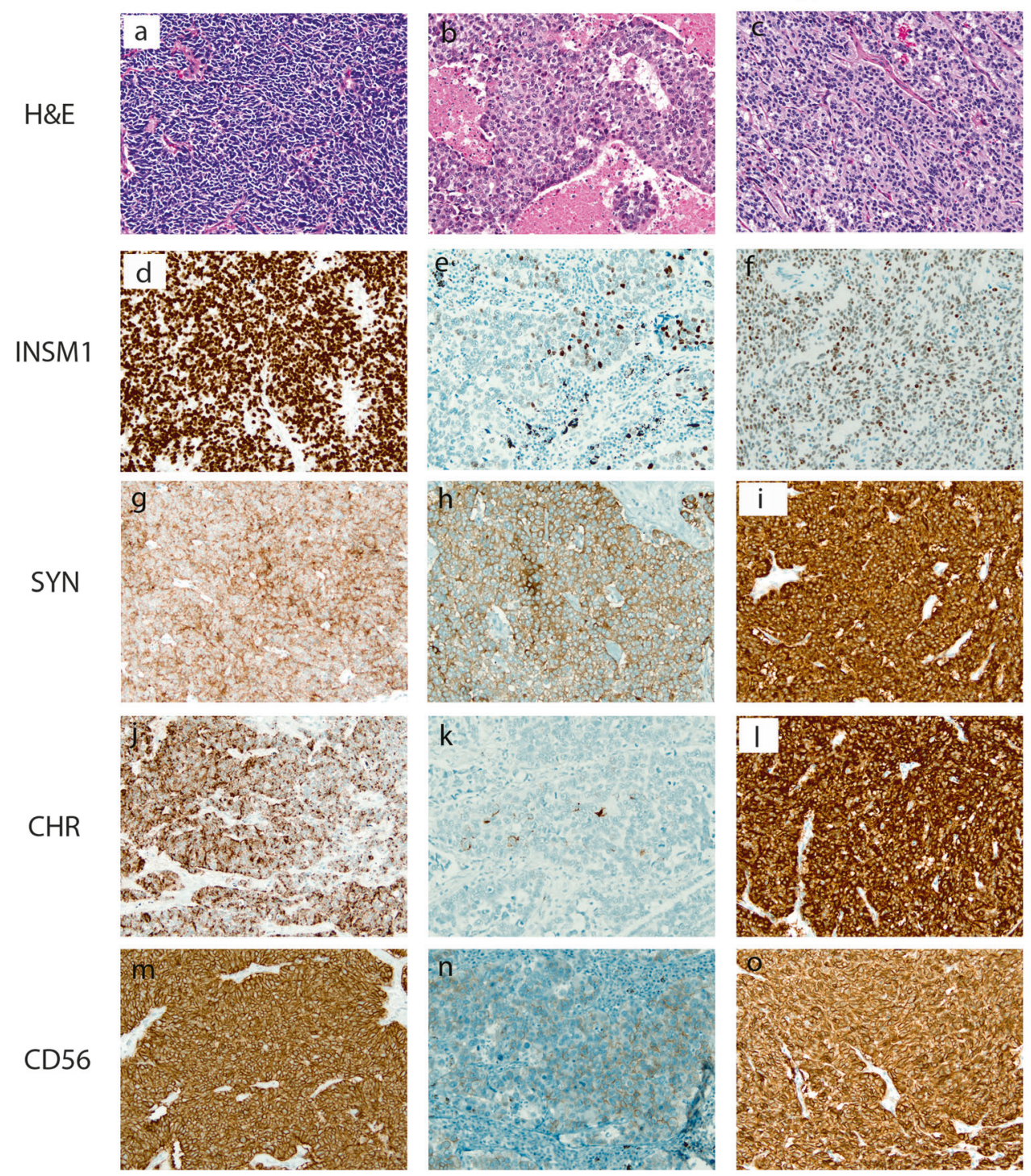

Table 4 Extent of

INSM1 staining in primary lung neoplasms

\begin{tabular}{llllll}
\hline & INSM1 pos/total & \multicolumn{4}{l}{ Percentage of positive tumor cell nuclei } \\
\cline { 3 - 6 } & & $\leq 10$ & $11-50$ & $51-90$ & $91-100$ \\
\hline Small cell lung carcinoma & $63 / 64$ & 10 & 14 & 23 & 16 \\
Large cell neuroendocrine carcinoma & $18 / 24$ & 11 & 4 & 2 & 1 \\
Typical carcinoid tumor & $48 / 48$ & 5 & 10 & 24 & 9 \\
Atypical carcinoid tumor & $15 / 16$ & 2 & 6 & 4 & 3 \\
Adenocarcinoma & $4 / 130$ & 4 & 0 & 0 & 0 \\
Squamous cell carcinoma & $1 / 33$ & 1 & 0 & 0 & 0 \\
\hline
\end{tabular}

to "smear out" or become granular. Table 5 shows various staining permutations in small cell lung carcinoma with the four neuroendocrine markers used in this study. Positive staining with all four markers was the most common scenario (51/64 cases, $80 \%)$, followed by positive staining with
INSM1, synaptophysin, and CD56, but negative staining for chromogranin (10/64 cases, 16\%). There were eight combined small cell lung carcinomas: six with a large cell neuroendocrine carcinoma component (small cell lung carcinoma-large cell neuroendocrine carcinoma), and two 
Table 5 Staining permutations with INSM1, synaptophysin, chromogranin, and CD56 in small cell lung carcinoma

\begin{tabular}{lllll}
\hline Small cell lung carcinoma (no. of cases) & INSM1 & Synaptophysin & Chromogranin & CD56 \\
\hline 51 & Positive & Positive & Positive & Positive \\
10 & Positive & Positive & Negative & Positive \\
2 & Positive & Positive & Positive & Negative \\
1 & Negative & Positive & Negative & Negative \\
\hline
\end{tabular}

Fig. 2 Variations in INSM1 staining in neuroendocrine lung neoplasms. a Patchy, weak INSM1 staining in small cell lung carcinoma. Most cells are negative. b INSM1 shows a "smeared" pattern in crushed area in small cell lung carcinoma (arrow), distinct from crisp nuclear staining in viable tumor cells (arrowhead). c Typical carcinoid tumor, with very minimal and focal INSM1 staining. d Very focal and weak INSM1 staining in an atypical carcinoid tumor. All images at original magnification $\mathrm{x} 200$

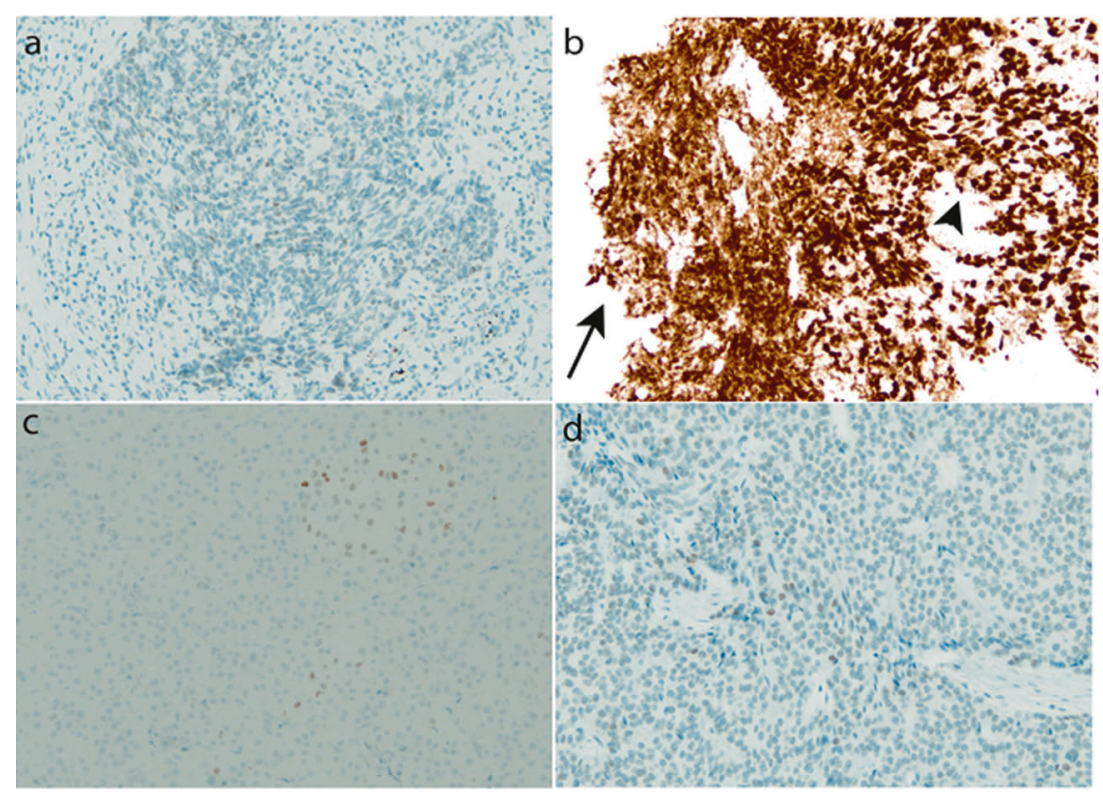

cases admixed with adenocarcinoma and squamous cell carcinoma, respectively. In 5/6 combined small cell lung carcinoma-large cell neuroendocrine carcinomas, both components stained with INSM1; in one case, only the small cell carcinoma component was positive. In the small cell lung carcinoma-squamous cell carcinoma and the small cell lung carcinoma-adenocarcinoma cases, the small cell lung carcinoma component was positive for INSM1 while the other component was negative (Fig. 2).

Of the 18 INSM1-positive large cell neuroendocrine carcinomas, only two (11\%) showed strong staining, the remainder being weak to moderate. A significant subset of cases $(11 / 18,61 \%)$ showed very focal staining $(\leq 10 \%)$ (Table 4 and Fig. 3).

Of the 48 typical carcinoid tumors, INSM1 staining was weak to moderate in 43 and strong in 5. Most (34/ $48,71 \%$ ) showed patchy staining in the $11-90 \%$ range, with intensity of staining ranging from weak to moderate to strong within the same neoplasm. Similarly, of the 15 INSM1-positive atypical carcinoids, strong INSM1 staining was seen in only two cases, the majority $(13,87 \%)$ showing weak to moderate staining. As with typical carcinoids, patchy staining in the $11-90 \%$ range accounted for most cases (Table 4). There was no significant difference in intensity or distribution of INSM1 staining between typical carcinoid tumors and atypical carcinoid tumors.

\section{INSM1 staining in non-neuroendocrine primary lung neoplasms}

Only $5(5 / 193,3 \%)$ non-neuroendocrine lung neoplasms stained with INSM1, including 4/130 (3\%) adenocarcinomas, and $1 / 33(3 \%)$ squamous cell carcinomas. In each of these tumor types, staining was very focal $(\leq 10 \%)$, and was noted on a whole-tissue section of a resected tumor (Fig. 3). The solitary squamous cell carcinoma that stained focally for INSM1 was also focally positive for CD56 but negative for synaptophysin and chromogranin. Of the four adenocarcinomas that were focally positive for INSM1, three were focally positive for synaptophysin and CD56 but negative for chromogranin; the fourth was negative for all three traditional neuroendocrine markers.

Of primary lung neoplasms whose INSM1 status has not been previously reported, including 30 non-neuroendocrine lung neoplasms of 8 histologic types, INSM1 was negative in all cases (Table 3). 
Fig. 3 INSM1 staining in a nonneuroendocrine neoplasm. a Adenocarcinoma, solid predominant (whole-tissue section of resected tumor, hematoxylin-eosin). The tumor was diffusely positive for TTF-1 and napsin A (not shown). b Focal strong INSM1 positivity. This was the only positive focus in the entire tumor. Both images are at original magnification $\times 200$

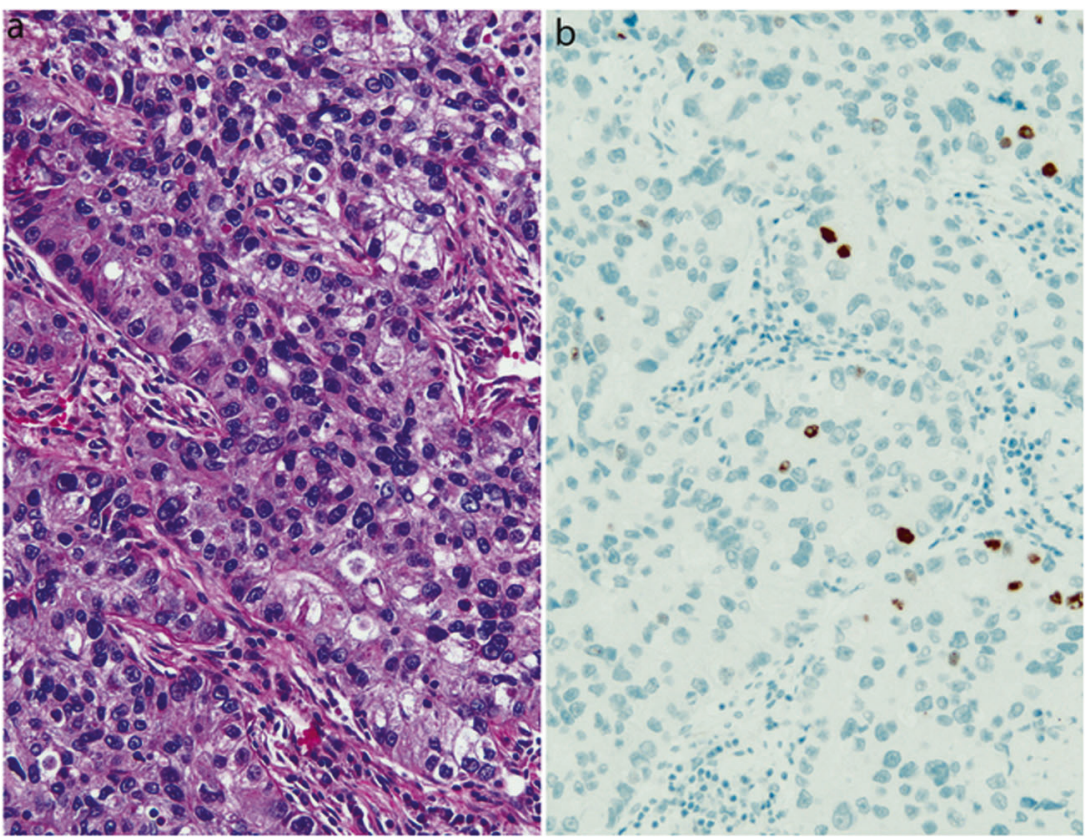

Table 6 Sensitivity and specificity of INSM1, synaptophysin, chromogranin and CD56 for neuroendocrine lung neoplasms

\begin{tabular}{llllll}
\hline & \multicolumn{2}{l}{ Positive/total (\%) } & & \\
\cline { 2 - 6 } & INSM1 & Synaptophysin & Chromogranin & CD56 & Any traditional neuroendocrine marker $^{\mathrm{a}}$ \\
\hline Sensitivity & $144 / 152(95)$ & $147 / 150(98)$ & $125 / 149(84)$ & $141 / 146(97)$ & $152 / 152(100)$ \\
Specificity & $188 / 193(97)$ & $38 / 42(90)$ & $119 / 121(98)$ & $106 / 122(87)$ & $28 / 46(61)$ \\
Positive predictive value & $144 / 149(97)$ & $147 / 151(97)$ & $125 / 127(98)$ & $141 / 157(90)$ & $152 / 170(89)$ \\
Negative predictive value & $188 / 196(96)$ & $38 / 41(93)$ & $119 / 143(83)$ & $106 / 111(95)$ & $28 / 28(100)$ \\
\hline
\end{tabular}

Sensitivity $=$ true positive/true positives + false negatives; specificity $=$ true negatives/true negatives + false positives; positive predictive value $=$ true positives/true positives + false positives; negative predictive value $=$ true negatives/true negatives + false negatives

${ }^{a}$ Synaptophysin, chromogranin or CD56

\section{Sensitivity and specificity of INSM1 for neuroendocrine lung neoplasms compared to traditional neuroendocrine markers}

The sensitivity of INSM1 for neuroendocrine lung neoplasms as a group (95\%) was similar to CD56 (97\%) and synaptophysin $(98 \%)$ and higher than chromogranin $(84 \%)$ (Table 6). For small cell lung carcinoma, the sensitivity of INSM1 (98\%) was similar to synaptophysin (100\%) and CD56 (95\%) and greater than chromogranin (83\%) (Table 3). For large cell neuroendocrine carcinoma, CD56 (92\%) and synaptophysin $(88 \%)$ were more sensitive than INSM1 (75\%) and chromogranin (46\%). In terms of specificity for neuroendocrine lung neoplasms, INSM1 (97\%) was similar to chromogranin $(98 \%)$ but higher than synaptophysin $(90 \%)$ or CD56 (87\%) (Table 6).

We also compared the performance of INSM1 as a neuroendocrine marker to the three traditional neuroendocrine markers as a group. For this analysis, staining for traditional neuroendocrine markers was considered positive if any traditional marker (synaptophysin, chromogranin, or CD56) was positive. Using this criterion, INSM1 was slightly less sensitive $(144 / 152,95 \%)$ than the traditional neuroendocrine markers as a group for all neuroendocrine neoplasms (152/ $152,100 \%)$. INSM1 was also marginally less sensitive for small cell lung carcinoma $(63 / 64,98 \%)$ compared to traditional neuroendocrine markers as a group $(64 / 64,100 \%)$. However, in terms of specificity, INSM1 (174/179, 97\%) was superior to traditional neuroendocrine markers as a group $(28 / 46,61 \%)$.

\section{INSM1 in primary tumors and matched metastases}

INSM1 staining in seven primary neuroendocrine neoplasms was compared to their matched metastases. In all cases, INSM1 was positive in the primary tumor as well as the matched metastasis (Fig. 4, Table 7). For non- 
Fig. 4 INSM1 staining in primary small cell lung carcinoma and matched cerebellar metastasis. Lung biopsy, (a) hematoxylin-eosin, original magnification $\times 200$. (b) INSM1 shows patchy positivity, original magnification $\times 200$. Cerebellar metastasis, $(\mathbf{c})$ hematoxylin-eosin, original magnification $\times 100$. (d) INSM1 is strongly and diffusely positive, original magnification $\times 100$. Cerebellar granular layer at bottom left is negative

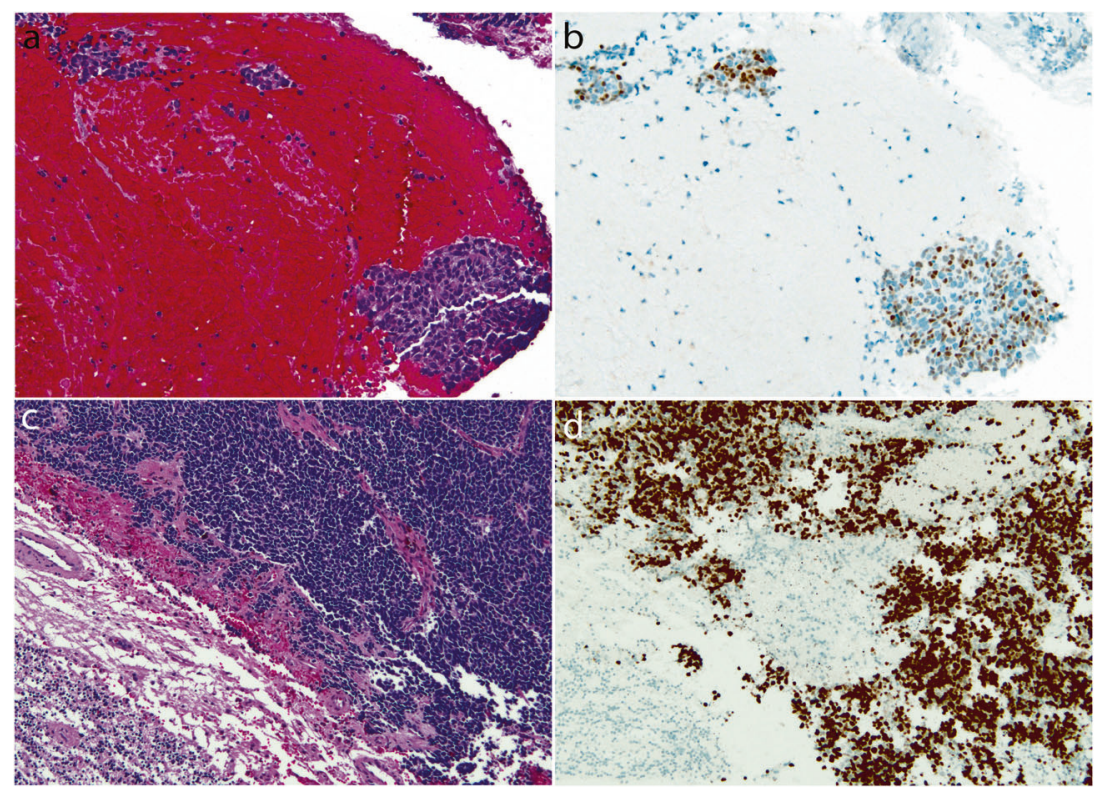

Table 7 INSM1 staining in matched metastases

\begin{tabular}{|c|c|c|c|c|c|}
\hline Diagnosis & $\begin{array}{l}\text { INSM1 in primary tumor } \\
\text { (result, intensity, \% of } \\
\text { positive neoplastic cells) }\end{array}$ & $\begin{array}{l}\text { H-score }{ }^{a} \\
(0-300)\end{array}$ & $\begin{array}{l}\text { INSM1 staining in matched } \\
\text { metastasis (result, intensity, } \% \text { of } \\
\text { positive neoplastic cells) }\end{array}$ & $\begin{array}{l}\text { H-score }{ }^{a} \\
(0-300)\end{array}$ & Site of metastasis \\
\hline Small cell lung carcinoma & Positive, weak, $90 \%$ & 90 & Positive, strong, $100 \%$ & 300 & Cerebellum \\
\hline Small cell lung carcinoma & Positive, weak, $75 \%$ & 75 & Positive, weak, 95\% & 95 & Brain \\
\hline Small cell lung carcinoma & Positive, strong, $100 \%$ & 300 & Positive, strong, $100 \%$ & 300 & Hilar lymph node \\
\hline $\begin{array}{l}\text { Large cell neuroendocrine } \\
\text { carcinoma }\end{array}$ & Positive, moderate, $10 \%$ & 20 & Positive, strong, 5\% & 15 & Lymph node \\
\hline $\begin{array}{l}\text { Small cell carcinoma of } \\
\text { pancreas }\end{array}$ & Positive, strong, $95 \%$ & 285 & Positive, strong, $100 \%$ & 300 & Lung \\
\hline Carcinoid tumor, lung & Positive, weak, $50 \%$ & 50 & Positive, weak, $20 \%$ & 20 & Hilar lymph node \\
\hline Carcinoid tumor, lung & Positive, moderate, $90 \%$ & 180 & Positive, moderate, $80 \%$ & 160 & Bone \\
\hline Adenocarcinoma & Negative & 0 & Negative & 0 & Hilar lymph node \\
\hline Adenocarcinoma & Negative & 0 & Negative & 0 & Hilar lymph node \\
\hline Adenocarcinoma & Negative & 0 & Negative & 0 & $\begin{array}{l}\text { Mediastinal } \\
\text { lymph node }\end{array}$ \\
\hline Adenocarcinoma & Negative & 0 & Negative & 0 & Hilar lymph node \\
\hline Adenocarcinoma & Positive, strong, $5 \%$ & 15 & Positive, strong, $5 \%$ & 15 & Hilar lymph node \\
\hline
\end{tabular}

${ }^{\mathrm{a}} \mathrm{H}$-score $=[1 \times(\%$ cells $1+)+2 \times(\%$ cells $2+)+3 \times(\%$ cells $3+)]$, with $0,1+, 2+$, or $3+$ assigned to a staining intensity of negative, weak, moderate, or strong.

neuroendocrine tumors, INSM1 staining in five primary tumors was compared to matched metastases. In four cases, both the primary and the metastasis were negative for INSM1. In one case (adenocarcinoma), the primary tumor showed focal INSM1 staining, which was also seen in the matched lymph node metastasis.

\section{INSM1 expression in normal lung tissue}

INSM1 staining of variable intensity was noted in occasional normal neuroendocrine cells (Kulchitsky cells) in bronchial and bronchiolar epithelium (Fig. 5). There was no INSM1 expression in alveolar pneumocytes, club cells, ciliated bronchial epithelial cells, or goblet cells.

\section{Discussion}

No new neuroendocrine markers have been introduced in diagnostic immunohistochemistry since 1990, when it was first recognized that CD56 could be used as a neuroendocrine marker in human tumors such as small cell carcinoma 
Fig. 5 INSM1 in normal lung. a INSM1 marks the nuclei of rare neuroendocrine (Kulchitsky) cells in bronchial epithelium (arrows, original magnification $\times 100)$. b Few INSM1-positive Kulchitsky cells in a normal bronchiole (arrows, original magnification $\times 200)$. Alveolar pneumocytes (bottom left) are negative

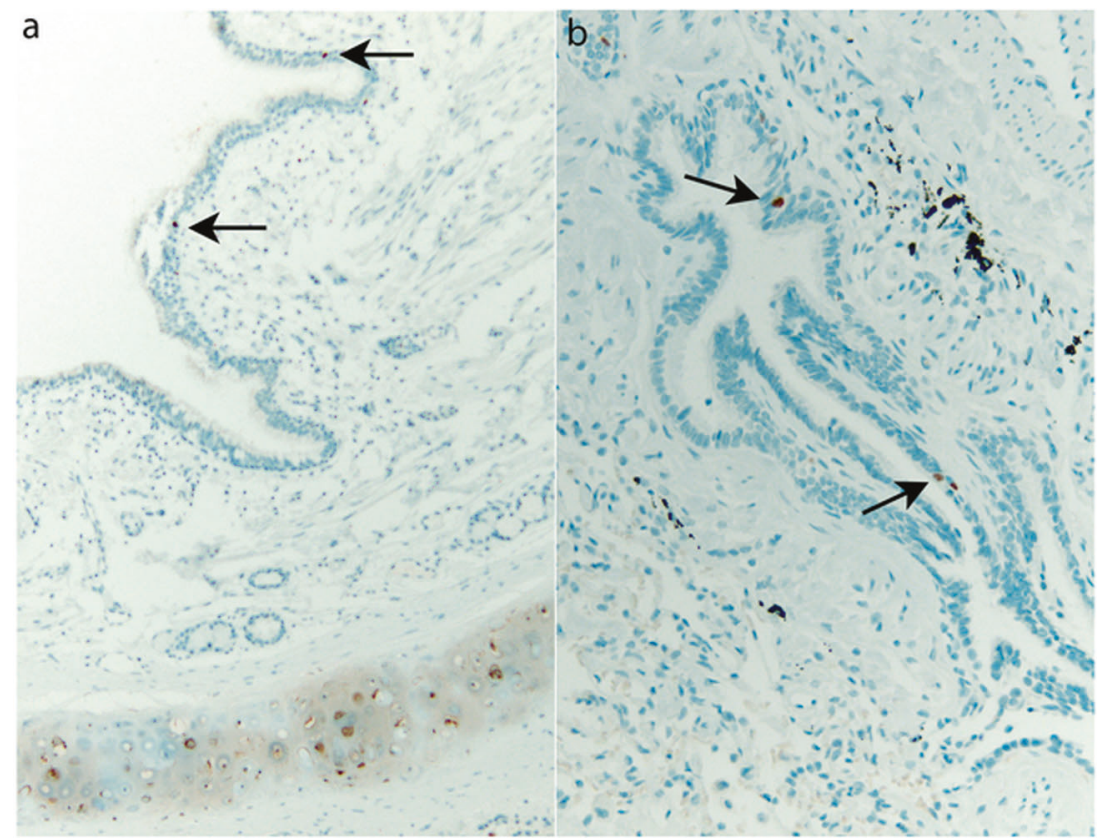

[22]. Although several antibodies against nuclear transcription factors have emerged as useful immunohistochemical markers in recent years, a nuclear marker of neuroendocrine differentiation has been elusive until recently. A sensitive and specific nuclear marker of neuroendocrine differentiation would be especially valuable in pulmonary pathology, where neuroendocrine tumors are commonly encountered. Although the diagnosis of neuroendocrine lung neoplasms is not difficult in classic cases, most of which can be diagnosed on the basis of standard histologic or cytologic stains without ancillary immunohistochemical testing [21], immunohistochemistry can be useful, especially when clinical or histologic features stray from the norm. The utility of neuroendocrine markers is perhaps greatest for high-grade neuroendocrine carcinomas. In small cell lung carcinoma, immunohistochemistry has been shown to improve diagnostic reproducibility, at least in challenging cases [23]. For large cell neuroendocrine carcinoma, a diagnosis that requires at least one positive neuroendocrine marker, all three currently available markers (synaptophysin, chromogranin, CD56) have limitations. Synaptophysin is sensitive but not entirely specific, chromogranin is highly specific but lacks sensitivity, and CD56 is highly sensitive but can stain a variety of nonneuroendocrine tumors, limiting its specificity.

The results of this study, the largest series of primary lung neoplasms stained with INSM1 to date, are mostly in agreement with those of prior smaller series. The sensitivity of INSM1 for small cell lung carcinoma in our study $(63 / 64$, $98 \%$ ) is comparable to data reported by Rooper et al. (37/ $39,94.9 \%)$, Fujino et al. (19/19, 100\%), and Rosenbaum et al. $(3 / 3,100 \%)$ in surgical pathology samples, as well as our previously reported results using Cellient cell blocks prepared from cytology samples $(38 / 41,93 \%)[11,12,14$, 19]. For carcinoid tumors, the sensitivity of INSM1 in our study $(63 / 64,98 \%)$ is equivalent to the $100 \%$ sensitivity reported by Rooper et al. (41/41), Rosenbaum et al. (6/6), and Fujino et al. (5/5) in surgical pathology, and our own findings in lung cytology samples $(9 / 10,90 \%)$.

For unclear reasons, the sensitivity of INSM1 for large cell neuroendocrine carcinoma in our study $(18 / 24,75 \%)$ is considerably lower than that reported by Rooper et al. (21/ $23,91.3 \%$ ). This area needs clarification in future studies. Another difference between the two studies is that the weak and focal INSM1 labeling that we observed in many carcinoid tumors contrasts with the more diffuse distribution and stronger staining intensity described by Rooper et al. This is difficult to explain, since we used the same clone (A8) from the same company (Santa Cruz), and both studies used citrate antigen retrieval. However, we did identify a few minor differences in methodology. The dilution of INSM1 in our study (1:250) was slightly lower than in the Rooper study (1:200), antibody was dispended manually in our study, and we used Ventana's Optiview detection kit with the optional amplifier, while Rooper et al. used Ventana's UltraView detection kit. Although the focal and weak staining seen in many carcinoid tumors in our study may be partially attributable to these technical differences, it remains unclear why these factors would cause differences in staining intensity and distribution only in some carcinoid tumors but not in small cell carcinomas.

The main advantage of INSM1 is its unique combination of high sensitivity and near-perfect specificity, which can justifiably be considered superior to any single traditional 
neuroendocrine marker. When INSM1 is compared to the traditional markers as a group, it is slightly less sensitive but considerably more specific. These characteristics have led some to suggest that INSM1 can be used as a standalone first-line marker of neuroendocrine differentiation [14], potentially helping to conserve tissue and reduce costs. Whether the imperfect sensitivity of INSM1 for small cell lung carcinoma is offset by its high specificity remains unresolved, and should be addressed in future studies.

The use of a large number of whole-tissue sections of resected tumors in this study provided insights into staining patterns that might be encountered with INSM1. The most important observation was that INSM1 staining can be very focal and weak not only in carcinoid tumors, but also in small cell lung carcinoma and large cell neuroendocrine carcinoma. This heterogeneity may be significant, since it implies that negative staining in a small biopsy does not exclude focal positivity in a resected tumor. Our finding that INSM1 staining is retained in metastatic sites is novel, and suggests that INSM1 expression in metastatic sites is a reliable surrogate for the INSM1 status of the primary tumor.

Despite the encouraging results, our findings do not provide compelling evidence to justify complete replacement of traditional neuroendocrine markers with INSM1. In carcinoid tumors, staining with traditional markers is highly sensitive and specific and easily interpretable, whereas INSM1 staining can be patchy and weak. In small cell lung carcinoma, traditional markers are easy to interpret in most cases and show stronger and more diffuse staining than INSM1 in many. INSM1 would be of great value if it was positive in small cell lung carcinomas that were negative for all three traditional neuroendocrine markers. However, we did not encounter a single instance of this situation in the present study. In addition, INSM1 is expressed in benign neuroendocrine cells and carcinoid tumorlets [17], and should not be used as a marker of neoplasia or malignancy in the lung. Also, INSM1 is expressed in neuroendocrine tumors of various primary sites and, therefore, it is not a marker of lung origin $[11,13,15,16,20]$. Finally, INSM1 does not help in the differential diagnosis between typical and atypical carcinoid tumors, low-grade and high-grade neuroendocrine carcinomas, or small cell lung carcinoma and large cell neuroendocrine carcinoma. Each of these neoplasms can be positive for INSM1, and the staining pattern overlaps too much to be discriminatory.

In summary, INSM1 is a reliable marker of neuroendocrine differentiation in lung neoplasms, with a sensitivity similar to synaptophysin and CD56, and specificity comparable to chromogranin. It may be used as a complementary marker to traditional neuroendocrine markers, just as the combination of nuclear and cytoplasmic markers (e.g., TTF-1 and napsin A) can occasionally be useful in other settings $[24,25]$. As a nuclear neuroendocrine marker, it may be easier to interpret in cases with few cells, extensive necrosis, marked crush artifact, or weak/equivocal staining with traditional neuroendocrine markers. Further investigation is required to define the precise circumstances where this stain would be most helpful.

Acknowledgements This study was supported by departmental funding.

\section{Compliance with ethical standards}

Conflict of interest The authors declare that they have no conflict of interest.

\section{References}

1. Goto Y, de Silva MG, Toscani A, et al. A novel human insulinoma-associated cDNA, IA-1, encodes a protein with "zincfinger" DNA-binding motifs. J Biol Chem. 1992;267:15252-7.

2. Lan MS, Russell EK, Lu J, et al. IA-1, a new marker for neuroendocrine differentiation in human lung cancer cell lines. Cancer Res. 1993;53:4129-440.

3. Xie J, Cai T, Zhang H, et al. The zinc-finger transcription factor INSM1 is expressed during embryo development and interacts with the Cbl-associated protein. Genomics. 2002;80:54-61.

4. Lan MS, Li Q, Modi WS, et al. Genomic organization, 5 upstream sequence, and chromosomal localization of an insulinomaassociated intronless gene, IA-1. J Biol Chem. 1994;269:14170-4.

5. Breslin MB, Zhu M, Notkins AL, et al. Neuroendocrine differentiation factor, IA-1, is a transcriptional repressor and contains a specific DNA-binding domain: identification of consensus IA-1 binding sequence. Nucleic Acids Res. 2002;30:1038-45.

6. Lan MS, Breslin MB. Structure, expression, and biological function of INSM1 transcription factor in neuroendocrine differentiation. FASEB J. 2009;23:2024-33.

7. Fujino K, Motooka Y, Hassan WA, et al. Insulinoma-associated protein 1 is a crucial regulator of neuroendocrine differentiation in lung cancer. Am J Pathol. 2015;185:3164-77.

8. Gierl MS, Karoulias N, Wende $\mathrm{H}$, et al. The Zinc-finger factor Insm1 (IA-1) is essential for the development of pancreatic $B$ cells and intestinal endocrine cells. Genes Dev. 2006;20:465-78.

9. Wildner H, Gierl MS, Strehle M, et al. Insm1 (IA-1) is a crucial component of the transcriptional network that controls differentiation of the sympatho-adrenal lineage. Development. 2008;135:473-81.

10. Ames HM, Rooper LM, Laterra JJ, et al. INSM1 expression is frequent in primary central nervous system neoplasms but not in the adult brain parenchyma. J Neuropathol Exp Neurol. 2018;77:374-82.

11. Rosenbaum JN, Guo Z, Baus RM, et al. INSM1: a novel immunohistochemical and molecular marker for neuroendocrine and neuroepithelial neoplasms. Am J Clin Pathol. 2015;144:579-91.

12. Fujino K, Yasufuku K, Kudoh S, et al. INSM1 is the best marker for the diagnosis of neuroendocrine tumors: comparison with CGA, SYP and CD56. Int J Clin Exp Pathol. 2017;10:5393-405.

13. Kuji S, Watanabe R, Sato Y. A new marker, insulinomaassociated protein 1 (INSM1) for high-grade neuroendocrine carcinoma of the uterine cervix: analysis of 37 cases. Gyn Oncol. 2017;144:384-90. 
14. Rooper LM, Sharma R, Li QK, et al. INSM1 demonstrates superior performance to the individual and combined use of synaptophysin, chromogranin and CD56 for diagnosing neuroendocrine tumors of the thoracic cavity. Am J Surg Pathol. 2017;41:1561-9.

15. Tanigawa $M$, Nakayama $M$, Taira $T$, et al. Insulinoma-associated protein 1(INSM1) is a useful marker for pancreatic neuroendocrine tumor. Med Mol Morphol. 2018;51:32-40.

16. Rush PS, Rosenbaum JN, Roy M, et al. Insulinoma-associated 1: a novel nuclear marker in Merkel cell carcinoma (cutaneous neuroendocrine carcinoma). J Cutan Pathol. 2018;45:129-35.

17. Dermawan JK, Mukhopadhyay S. Insulinoma-associated protein 1 (INSM1) differentiates carcinoid tumorlets of the lung from pulmonary meningothelial-like nodules. Histopathology. 2018;72:1067-9.

18. Yoshida A, Makise N, Wakai S, et al. INSM1 expression and its diagnostic significance in extraskeletal myxoid chondrosarcoma. Mod Pathol. 2018;31:744-52.

19. Doxtader EE, Mukhopadhyay S. Insulinoma-associated protein 1 is a sensitive and specific marker of neuroendocrine lung neoplasms in cytology specimens. Cancer Cytopathol. 2018;126:243-52.

20. Rooper LM, Bishop JA, Westra WH. INSM1 is a sensitive and specific marker of neuroendocrine differentiation in head and neck tumors. Am J Surg Pathol. 2018;42:665-71.
21. Brambilla E, Beasley MB, Austin JHM, et al. Neuroendocrine tumours. Small cell carcinoma. In: Travis WD, Brambilla E, Burke AP, et al. eds. WHO classification of tumours of the lung, pleura, thymus and heart. 4th ed. Lyon, France: IARC; 2015. p. 63-8.

22. Koros AM. Neuroendocrine markers expressed by human natural killer (NK) cells are also detectable on human small cell carcinomas, neuroblastomas, and sea urchin coelomocytes. Int J Neurosci. 1990;51:255-6.

23. Thunnissen E, Borczuk AC, Flieder DB, et al. The use of immunohistochemistry improves the diagnosis of small cell lung cancer and its differential diagnosis. An International Reproducibility Study in a challenging set of cases. J Thorac Oncol. 2017;12:334-46.

24. Mukhopadhyay S, Katzenstein AL. Comparison of monoclonal napsin A, polyclonal napsin A and TTF-1 for determining lung origin in metastatic adenocarcinomas. Am J Clin Pathol. 2012;138:703-11.

25. Mukhopadhyay S, Katzenstein AL. Subclassification of non-small cell lung carcinomas lacking morphologic differentiation on biopsy specimens: utility of an immunohistochemical panel containing TTF-1, napsin A, p63 and CK5/6. Am J Surg Pathol. 2011;35:15-25. 